\title{
FINITE ELEMENT MODELING OF UPSETTING CONSIDERING HEAT GENERATION
}

\author{
Roland MIHALOVITS 1 , Viktor GONDA², Károly SZÉLL ${ }^{3}$ \\ ${ }^{1,2}$ Óbuda University, Bánki Donát Faculty of Mechanical and Safety Engineering, Institute of Materials and \\ Manufacturing Sciences \\ ${ }^{1}$ mihalovits.roland@gmail.com \\ 2 gonda.viktor@bgk.uni-obuda.hu \\ ${ }^{3}$ Óbuda University, Regia Alba Technical Faculty, Székesfehérvár, Hungary, szell.karoly@amk.uni-obuda.hu
}

\begin{abstract}
In this article, a finite element modeling of the upsetting of a cylindrical workpiece is presented. In the modeling, the effects of friction, heat generation from deformation, and heat losses from conduction and convection are considered.
\end{abstract}

Keywords: finite element analysis, coupled model, friction, thermal balance.

\section{Introduction}

With the finite element method (FEM), the technological design of metal forming can be greatly simplified. Calculation of technological parameters in complex shaping cases requires very complicated formulas, or can be estimated with great simplifications, their measurement cannot be solved or is costly. The finite-element method can provide a solution in such cases, with the help of simulations, virtual prototyping can also shorten the trial production process, therefore significant cost savings can be achieved. With the help of the model, the changes of tensions, deformations and other parameters that can be calculated from these can be studied during the forming process, thus helping to optimize the technology [1].

FEM is a numerical method by which the mechanical and/or thermal model of the bodies is not constructed as a continuum, but is rather divided into parts, so-called elements. This method, in addition to today's available computing capacity, significantly accelerates design and calculation times.

For finite element modelling, we used MSC Marc Mentat 2016, which is well suited for the ther- mal-mechanical design of metal forming technologies [2].

In this paper, we describe the finite element modelling of a cylindrical workpiece, where the aim is to employ a mechanical-plastic model, which is further extended with thermal coupling, to investigate heat build-up due to rapid deformation, and to calculate cooling after forming. The modelling is divided into several levels of detail from the initial friction-free basic model to a model that is suitable for conducting thermal tests.

\section{Structure of the model}

The steel cylinder workpiece dimensions are $\emptyset 20 \mathrm{~mm}$ diameter and $30 \mathrm{~mm}$ in height. When constructing the model, only a quarter was drawn exploiting symmetry, with regard to a planar axis-symmetric case. The diameter of the tool is $\emptyset 30$ $\mathrm{mm}$ and the height is $10 \mathrm{~mm}$. During the upsetting, the moving tool part is displacement-controlled, the movement is linear, while the forming stroke is one third of the workpiece height.

The complexity of the model was divided into four levels: the first level model belongs to the ideal formulation, in the second level the friction is taken into account, for the third level the heat 
development, and for the fourth level the thermal conductivity of the tool is involved.

The tool is modeled as rigid in the first level model, therefore meshing is not necessary. An elastic-plastic hardening material model is employed, therefore meshing is necessary. The contact is considered frictionless.

After the geometries are completed, the next step is to specify the mechanical properties of the material (Table 1.), to establish contacts, the load case and to set the calculation job. With the successful running of the simulation, the basic model is appropriate, the basic model is suitable for validating the correctness of the main components and the running of the calculation job.

The second modelling level is friction consideration. Not only the value of the coefficient of friction but also the type of friction should be used, in this model we used Kudo friction. The Kudo friction number is defined as $\mathrm{m}=0.8$ between the workpiece and the tool.

The third modelling level is the examination of heat development due to rapid deformation. For this purpose, the thermal properties of the workpiece had to be defined: thermal conductivity, specific heat capacity (specific heat) and initial temperature of $293 \mathrm{~K}$. The additional thermal and mechanical parameters are shown in Table 1. In addition, the use of the thermal coupling of the model due to plastic deformation has to be activated.

Table 1. The thermal and mechanical parameters

\begin{tabular}{|c|c|c|}
\hline Property & Workpiece & Die \\
\hline Young's modulus [GPa] & 200 & - \\
\hline Poisson's ratio [-] & 0.3 & - \\
\hline Yield strength [MPa] & 275 & - \\
\hline Hardening coefficient [MPa] & 722 & - \\
\hline Hardening exponent [-] & 0.262 & - \\
\hline Density $\left[\mathrm{kg} / \mathrm{m}^{3}\right]$ & \multicolumn{2}{|c|}{7800} \\
\hline Heat conduction coeff. $\left[\frac{W}{m \cdot K}\right.$ & 36 & 40 \\
\hline Spec. heat capacity $\left[\frac{J}{m^{2} \cdot K}\right]$ & 450 & 350 \\
\hline $\begin{array}{l}\text { Heat transf. coeff. }\left[\frac{W}{m^{2} \cdot K}\right] \\
\text { (between environment and } \\
\text { workpiece) }\end{array}$ & \multicolumn{2}{|c|}{2.95} \\
\hline $\begin{array}{l}\text { Heat tranfs. coeff. }\left[\frac{W}{m^{2} \cdot K}\right] \\
\text { (between workpiece and die) }\end{array}$ & \multicolumn{2}{|c|}{400} \\
\hline
\end{tabular}

At the fourth model level, the thermal conductivity of the tool is also incorporated so that changes in heat balance during and after the operation can be investigated. Therefore, in this case, there is a need for a meshed die, since the program can only calculate different parameters for nodes, i.e. the temperature, but consider the tool mechanically as rigid, so it will be a rigid model. We also defined the heat transfer coefficient between the workpiece and the tool and the environment whose values are also shown in Table 1 . When modifying and supplementing existing models, care must be taken, since overlapping nodes of each contact can easily distort the entire model, since duplicate nodes and elements must be deleted after modelling.

\section{Results}

The results of the different modelling levels are presented separately. The first-level basic model is designed to check the correct run, so it's not being detailed right now.

\subsection{The effect of friction}

The effect of friction appears in the barreling of the workpiece, as shown in Figure 1. In the figure, the axis of symmetry is the horizontal axis, the surface of the workpiece contacting the tool is the left vertical line in this section and the right line represents the plane of mirror symmetry.

The barreling can be explained by the frictional force on the workpiece and on the tool's surface

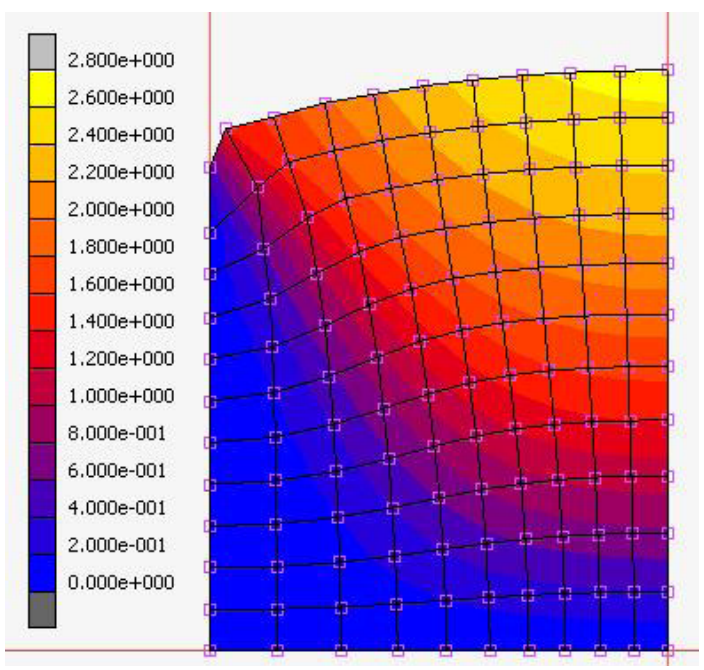

Figure 1. The phenomenon of barreling ( $m=0.8)$. The scale shows the radial displacement in $\mathrm{mm}$ 
that prevents movement in the radial direction so that the diameter increase in these positions during the upsetting will be smaller. As we move axially from the tool, the effect of this phenomenon is reduced.

Increasing the value of friction coefficient does not always result in a larger radial increase in size.

As can be seen in Figure 1., the high coefficient results in the first element suffering from a very large distortion, with a significant leap in the curvature. This can be eliminated by denser meshing in order to obtain better results.

\subsection{Heat generation}

The deformation results in heat generation in the workpiece. If the forming is fast enough, in this case, 0.5 seconds, then the adiabatic case can be evaluated by neglecting the heat loss. The workpiece temperature rises, the temperature distribution is not homogeneous, as can be seen in Figure 2. The highest temperature is at the centre of the workpiece until the lowest temperature at the point of the workpiece axis at the plane of the tool plane, here the friction has no significant effect. The maximum temperature resulting from deformation is 380 Kelvin, which is 87 Kelvin higher than the initial room temperature.

\subsection{Heat loss}

The heat balance should be inspected with the stamp position at the end of the forming so that

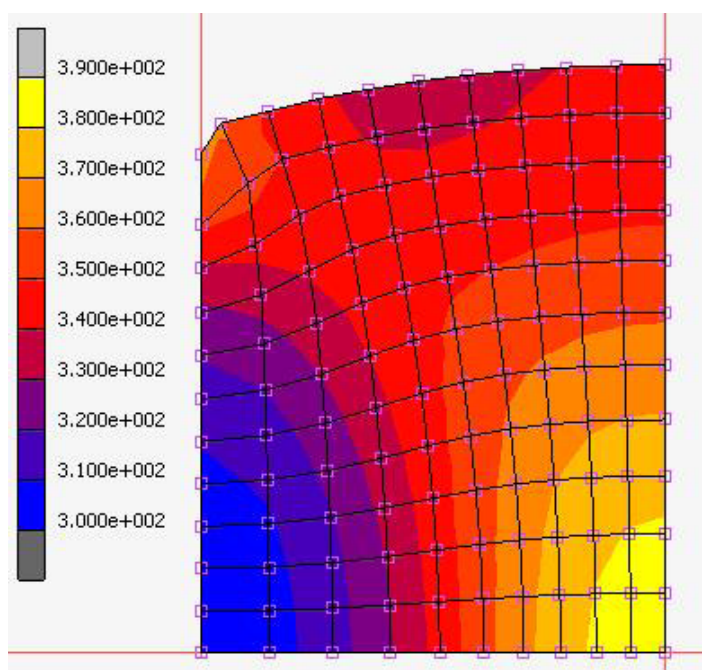

Figure 2. The temperature distribution at the end of the deformation the effect of the thermal conductivity of the tool can be demonstrated.

In order to evaluate the results at the different time points $(\mathrm{t}=\{0,5 ; 1,82 ; 10,26 ; 43,46 ; 100,5\} \mathrm{s})$, the temperature from the upper plane of the tool to the half-height of the workpiece was added.

The results thus obtained have been combined in the graph in Figure 3. to observe that at the end of the forming ( $t=0.5 \mathrm{~s}$ ) the highest temperature is near the contact plane of the tool and workpiece since there was the largest deformation. Then, in the end, position, the temperature from this first node began to spread in both directions since the temperature of this node decreased while the temperature of the tool or adjacent nodes increased, this phenomenon can be observed at $\mathrm{t}=1.82 \mathrm{~s}$.

Then, the workpiece and the tool cool down this heat distribution characteristic after 100 seconds of initial temperature.

\section{Summary}

The finite element model of the upsetting was completed with four different levels of detail to investigate the effect of friction, heat build-up due to rapid deformation and the resulting heat dissipation.

\section{Acknowledgements}

The authors express their thanks to the Ministry of Human Resources ÚNKP-17-I-OE-779/15 grant support and the EFOP-3.6.1-16-2016-00010 tender support.

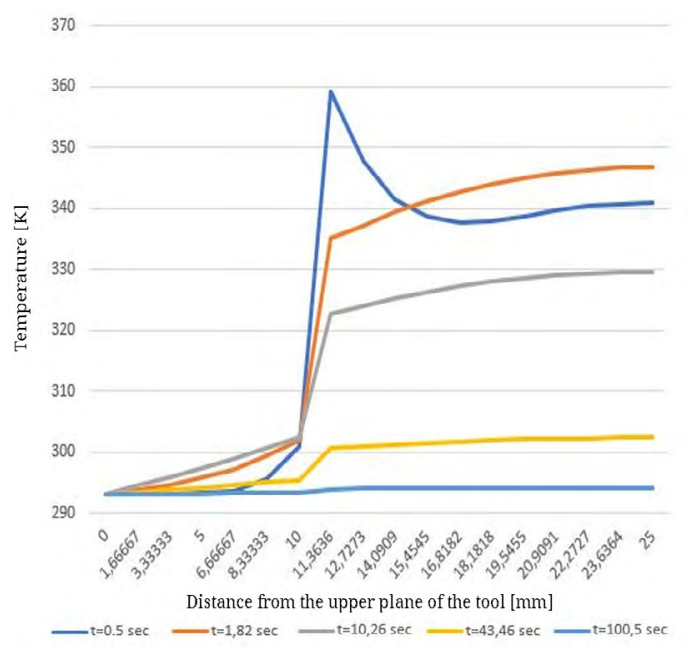

Figure 3. Temperature changes from the upper plane of the tool to the half-height of the workpiece 


\section{References}

[1] Henry S. Valberg: Applied Metal Forming, Chapter 11., Cambridge, 2010.

[2] Marc E: Demonstration Problems, Chapter 8.59; Volume MSc Software, 2013. 